J. Lake Sci. (湖泊科学), 2021, 33(1): 111-122

DOI 10. 18307/2021. 0125

(c) 2021 by Journal of Lake Sciences

\title{
夏季东太湖光合有效辐射衰减特征及其对沉水植物恢复的指示”
}

\author{
徐德瑞 ${ }^{1}$, 周 杰 $^{1,2 * *}$, 吴时强 ${ }^{1,2}$, 戴江玉 ${ }^{1}$, 吴修锋 ${ }^{1,2}$ \\ ( 1 : 南京水利科学研究院,南京 210029) \\ $(2:$ 水文水资源与水利工程科学国家重点实验室, 南京 210098)
}

\begin{abstract}
摘 要: 水下光照分布是影响水生态系统的重要因素, 研究光合有效辐射衰减特征对于沉水植物恢复具有一定的指导意 义. 根据沉水植物生物量资料, 将东太湖划分为沉水植物茂盛区、沉水植物稀疏区和无植物区 3 种区域. 基于 2019 年夏季 原位水下光场资料, 探讨了东太湖光衰减特性和光照衰减因子的空间差异以及不同区域内的主导衰减因子, 分析了东太 湖的稳态阶段和富营养化水平,并阐述了真光层深度与透明度的关系, 以期为东太湖沉水植物恢复和保护提供相关资料. 结果表明: 东太湖不同区域光衰减特性差异显著, 光合有效辐射衰减系数 $\left(k_{\mathrm{d}}(\mathrm{PAR})\right)$ 在 $0.73 \sim 11.80 \mathrm{~m}^{-1}$ 之间变化, 真光层 深度范围为 $0.39 \sim 6.31 \mathrm{~m}$. 不同区域的无机悬浮物和有机悬浮物浓度存在显著性差异, 稀疏区叶绿素 $a$ 浓度显著高于茂 盛区, 而与无植物区没有显著差异, 有色可溶性有机物 (CDOM) 吸收系数在 3 种区域无显著性差异. $k_{\mathrm{d}}(\mathrm{PAR})$ 与无机、有 机悬浮物的线性拟合效果较好, 而与叶绿素 $a 、$ CDOM 拟合较差. 水体吸收和散射作用是茂盛区光衰减的主要原因, 无植 物区域主导衰减因子仅有无机悬浮物, 稀疏区由叶绿素 $a$ 和无机悬浮物共同主导, 是生态修复需要重点关注的区域,有机 悬浮物和 CDOM 对东太湖光照衰减没有太大影响. 东太湖目前正处于从草型稳态向藻型稳态过渡的阶段, 整个湖泊属于 富营养水平,真光层深度大约为透明度的 2.7 倍.
\end{abstract}

关键词: 东太湖;光合有效辐射衰减系数;空间差异;主导因子;稳态转换;富营养化

\section{Attenuation characteristics of photosynthetically active radiation in summer eastern Lake- Taihu and implications for submerged plants restoration *}

\author{
Xu Derui ${ }^{1}$, Zhou Jie ${ }^{1,2 * *}$, Wu Shiqiang ${ }^{1,2}$, Dai Jiangyu ${ }^{1} \&$ Wu Xiufeng ${ }^{1,2}$ \\ (1: Nanjing Hydraulic Research Institute, Nanjing 210029, P.R. China) \\ (2: State Key Laboratory of Hydrology-Water Resources and Hydraulic Engineering, Nanjing 210098,P.R.China)
}

\begin{abstract}
The underwater light distribution is an important factor affecting aquatic ecosystems. The study on the attenuation characteristics of photosynthetically active radiation is of guiding significance for the restoration of submerged plants. In this study, based on the biomass data of submerged plants, eastern Lake Taihu was divided into submerged plants sparse region, submerged plants lush region, and no-plant region. Spatial difference in light attenuation characteristics and light attenuation factors of eastern Lake Taihu, dominant attenuation factors in different regions, regime shift phases and eutrophication level, and the relationship between euphotic depth and transparency were studied by applying in situ measurement data in August 2019. Results were analyzed to provide relevant optical data for the restoration and protection of submerged plants in eastern Lake Taihu. The results showed that the light attenuation characteristics are significantly different in three regions. The diffuse attenuation of photosynthetically active radiation varied from $0.73 \mathrm{~m}^{-1}$ to $11.80 \mathrm{~m}^{-1}$ and the corresponding euphotic depth was $0.39-6.31 \mathrm{~m}$. There were significant differences in the concentrations of suspended particulate inorganic matter and suspended particulate organic matter in different regions. The concentration of chlorophyll- $a$ in the sparse region was significantly higher than that in the lush region, but not significantly different from that in no-plant region. Chromophoric dissolved organic matter ( CDOM) absorption coefficient had no significant difference in three regions. The linear fitting effect of $k_{\mathrm{d}}$ (PAR) with suspended particulate inorganic matter and suspended particulate
\end{abstract}

* 2020-02-25 收稿; 2020-05-18 收修改稿.

国家水体污染控制与治理科技重大专项 (2017ZX07203-002)、江苏省水利科技项目 (2018006,2018001) 和国家自 然科学基金项目 (51479121) 联合资助.

** 通信作者;E-mail:jzhou@ nhri.cn. 
organic matter was better, but it was worse with chlorophyll-a and CDOM. Water absorption and scattering were the main cause of light attenuation in the lush region and suspended particulate inorganic matter was the dominant attenuation factor in no-plant region. The sparse region was dominated by chlorophyll- $a$ and suspended particulate inorganic matter, which was a region that needs to be focused on for ecological restoration. Suspended particulate organic matter and CDOM had little effect on the light attenuation. Eastern Lake Taihu was currently in the transition stage from a macrophytes-dominated state to a phytoplankton-dominated state, and the entire lake was at eutrophic level. Euphotic depth was about 2.7 times of the transparency.

Keywords: Eastern Lake Taihu; diffuse attenuation coefficient of photosynthetically active radiation; spatial difference; major influential factors; regime shift; eutrophication

湖泊水体的透光强度决定了水中生物的生长状态, 透光深度直接影响了沉水植物的定植深度. 沉水植 物恢复是生态修复的关键,也是水体生物多样性赖以维持的基础. 研究表明,水下光照强度对沉水植物生长 起着主要限制作用, 是影响沉水植物生长的主要环境因子 ${ }^{[1-2]}$, 研究水下光照衰减可为沉水植物生长提供一 定理论依据. 光照进人水体中会受到纯水、悬浮颗粒、浮游植物的吸收和散射以及有色可溶性有机物的吸收 作用, 从而引起光照的衰减 ${ }^{[3]}$. 早在 1970s, 国外学者就研究了悬浮颗粒、叶绿素 $a$ 对水下光照衰减的影 响 ${ }^{[4]}$, 到了 1990s, 我国开始研究太湖悬浮质、可溶性有机质和藻类色素对可见光的吸收和散射作用 ${ }^{[5]}$. 随后 杨顶田等 ${ }^{[6]}$ 、张运林等 ${ }^{[7]}$ 继续在太湖开展相关研究工作.

东太湖是太湖东部的一个湖湾, 水质曾是整个太湖最好的区域, 2002 年以前沉水植物分布广泛, 之后开 始减少, 近几年沉水植物生物量和分布面积都有所下降 ${ }^{[8-10]}$. 因此, 了解水体光衰减特性与主导因子, 有助于 探索改善光照环境的有效措施, 恢复沉水植物, 促进水生态系统的良性循环. 虽然东太湖的光学特性方面已 开展了相关研究 ${ }^{[7,11]}$, 但针对不同区域光衰减特性的详细研究未见报道. 不同水体环境中, 影响光衰减的主 要因素有所区别. 太湖是一个常年受风浪影响的浅水湖泊, 悬浮颗粒浓度较高. 相关研究表明,一般情况下 风浪扰动引起水中悬浮物增加是改变水下光照分布的主要原因, 但东太湖水生植物分布广泛, 湖湾较多, 波 浪影响小, 影响水下光照衰减的主导因素较为复杂. 另外, 以往大多只是关注如何恢复沉水植物, 而在草藻 共生区域保护现存沉水植物不受破坏更为重要. 因此, 本文基于 2019 年 8 月份的采样数据, 根据沉水植物 生物量划分区域, 旨在分析东太湖夏季不同区域的水体光学特征以及影响光照衰减的主导因素, 以期为保 护和恢复东太湖沉水植物以及类似湖泊研究提供基础资料.

\section{1 研究区域与方法}

\section{1 研究区域}

东太湖 $\left(30^{\circ} 58^{\prime} \sim 31^{\circ} 07^{\prime} \mathrm{N}, 120^{\circ} 25^{\prime} \sim 120^{\circ} 35^{\prime} \mathrm{E}\right)$ 是太湖东南部东山半岛东侧的一个湖湾, 与西太湖之间 以狭窄的湖面相通, 其末梢一直延伸到瓜泾口, 总长度 $27.5 \mathrm{~km}$, 最大宽度 $9.0 \mathrm{~km}$, 总面积 $131.25 \mathrm{~km}^{2}$, 平均水 深不超过 $1.2 \mathrm{~m}^{[12]}$.

本次研究共设置 10 个采样点, 如图 1 所示, 其中 $1^{\#} 、 6^{\#} 、 7^{\#}$ 采样点位于东山沿岸区域, $2^{\#} 、 5^{\#} 、 8^{\#} 、 10^{\#}$ 采样 点位于围网拆除区域, $3^{\#} 、 4^{\#} 、 9^{\#}$ 采样点位于行洪通道区域.

\section{2 样品采集与测定}

2019 年夏季 8 月份共 16 次对东太湖水深 $(H)$ 、透明度 $(\mathrm{SD})$ 、水下光合有效辐射 (PAR)、无机悬浮物 (ISS)、有机悬浮物 (OSS)、叶绿素 $a(\mathrm{Chl} . a)$ 和 CDOM 吸收系数等指标进行了调查, 采样期间无风或微风, 采 样时间控制在 $9: 00-12: 00$.

1.2.1 现场指标测定 水深使用测深杆进行测量, 透明度使用塞氏盘测量. 采用美国 Apogee 公司生产的 MQ510 全光谱水下光量子测量仪 (光谱响应范围为 $389 \sim 692 \mathrm{~nm} \pm 5 \mathrm{~nm}$, 测量范围为 $0 \sim 4000 \mu \mathrm{mol} /\left(\mathrm{m}^{2} \cdot \mathrm{s}\right.$ )), 测 量水体表面以下 $0 、 0.1 、 0.2 、 0.3 、 0.4 、 0.5 、 0.6 、 0.7 、 0.8 、 0.9 、 1.0 、 1.2 、 1.4 \mathrm{~m}$ 处的光照强度, 每层记录 3 个数据, 3 个数据的平均值作为该层的 PAR 光照强度.

1.2.2 实验室测定 在现场用 $1 \mathrm{~L}$ 聚乙烯塑料瓶取 $0.5 \mathrm{~m}$ 处表层水装满, 带回实验室储存在 $4^{\circ} \mathrm{C}$ 冰箱中, 并在 $48 \mathrm{~h}$ 内完成进行实验室分析测试. ISS OSS、Chl. $a$ 、总氮 (TN) 和总磷 (TP) 浓度等按照《湖泊富营养化调查规 范 $\rangle^{[13]}$ 进行测定. 


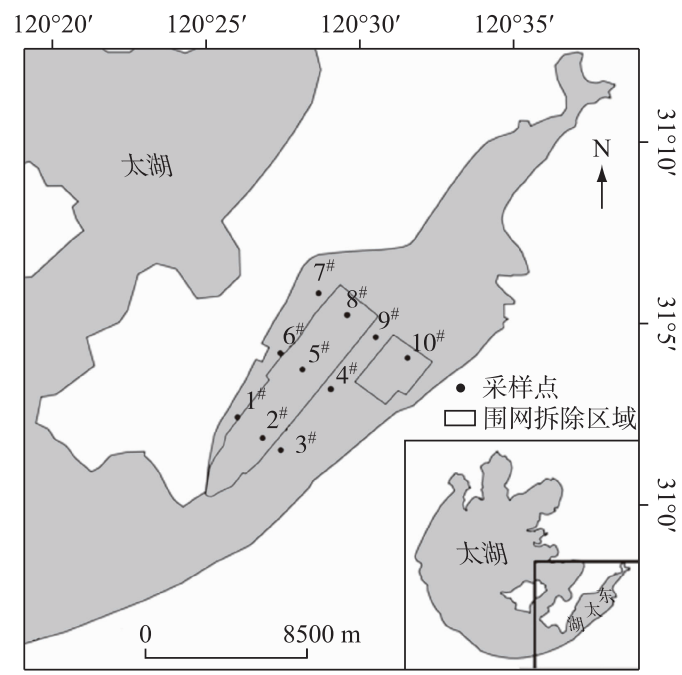

图 1 东太湖采样点分布

Fig.1 Location of sample sites in eastern Lake Taihu

有色可溶性有机物 (CDOM) 吸收系数测定时先用滤膜过滤水样, 将滤液再用 $0.22 \mu \mathrm{m}$ 的 Millipore 膜过 滤, 最后得到的滤液在 UV-2401 分光光度计下测定其吸光度, 然后根据式 (1) 计算出各波长的吸收系数, 并 用式(2) 进行散射校正 ${ }^{[14]}$.

$$
\begin{gathered}
\alpha_{\mathrm{CDOM}}\left(\lambda^{\prime}\right)=2.303 D(\lambda) / r \\
\alpha_{\mathrm{CDOM}}(\lambda)=\alpha_{\mathrm{CDOM}}\left(\lambda^{\prime}\right)-\alpha_{\mathrm{CDOM}}(750) \cdot \lambda / 750
\end{gathered}
$$

式中, $\alpha_{\mathrm{CDOM}}\left(\lambda^{\prime}\right)$ 为未校正的吸收系数, $\mathrm{m}^{-1} ; D(\lambda)$ 为吸光度; $r$ 为光程路径, $\mathrm{m} ; \lambda$ 为波长, $\mathrm{nm} ; \alpha_{\mathrm{CDOM}}(\lambda)$ 为 经过散射校正的吸收系数, $\mathrm{m}^{-1} ; \alpha_{\mathrm{CDOM}}(750)$ 为 $750 \mathrm{~nm}$ 处测定的吸收系数, $\mathrm{m}^{-1}$. 由于 CDOM 物质组成极为 复杂,本次研究用 $440 \mathrm{~nm}$ 吸收系数来表示其浓度.

1.2 .3 沉水植物生物量 使用水草采集镰刀 (用长为 $0.12 \mathrm{~m}$ 的刀头绑在 $3 \mathrm{~m}$ 竹竿上) 靠近底泥旋转一周, 采 集 $0.045 \mathrm{~m}^{2}$ 的沉水植物地上部分. 同一采样点采集 2 次以保证样品采集完全. 采集后用湖水清洗,去除泥、 枯枝败叶和其他杂质,称其鲜重. 所有称量操作均进行两次, 取其平均值记录.

\section{3 东太湖区域划分方法}

采样期间调查了各采样点沉水植物生物量和优势种. 根据生物量的大小初步将东太湖划分为 3 种区 域:沉水植物茂盛区、沉水植物稀疏区和无植物区.

\section{4 光衰减系数和真光层深度计算}

在均质和充分混合的水柱中, $k_{\mathrm{d}}(\mathrm{PAR})$ 值会随着深度而发生变化. $k_{\mathrm{d}}(\mathrm{PAR})(z)$ 是深度 $z$ 处的漫射衰减 系数: $k_{\mathrm{d}}(\mathrm{PAR})(z)=-\frac{\mathrm{d}(\ln (E(z)))}{\mathrm{d} z}$, 但大部分学者采用一种简化的模式, 即认为 $k_{\mathrm{d}}(\mathrm{PAR})$ 在观测深度范围 内不随深度变化, 而是在表面和深度 $z$ 之间取 $k_{\mathrm{d}}(\mathrm{PAR})$ 的垂直平均值, 从而得到光衰减系数的计算式 ${ }^{[15]}$ :

$$
k_{\mathrm{d}}(\mathrm{PAR})=-\frac{\ln (E(z))-\ln (E(0))}{z}
$$

式中, $z$ 为水面到测量处的深度, $E(z)$ 为深度 $z$ 处的辐照度, $E(0)$ 为表面辐照度. 真光层深度为辐照度为水 体表面辐照度 $1 \%$ 的深度, 常作为恢复沉水植物的临界深度 ${ }^{[11]}$,计算公式为：

$$
D_{\text {eu }}=-\frac{\ln (E(z))-\ln (E(0))}{k_{\mathrm{d}}(\mathrm{PAR})}=-\frac{1}{k_{\mathrm{d}}(\mathrm{PAR})} \ln \frac{E(0) \times 1 \%}{\mathrm{E}(0)}=4.605 / k_{\mathrm{d}}(P A R)
$$

式中, $D_{\text {eu }}$ 为真光层深度, $\mathrm{m} ; k_{\mathrm{d}}(\mathrm{PAR})$ 为光合有效辐射衰减系数. 


\section{5 草型、藻型湖泊划分依据和富营养化评价方法}

根据 Bachmann 等方法对藻型稳态和草型稳态进行划 ${ }^{\text {分 }}{ }^{[16]}$ : 草藻干重比 $a=$ 沉水植物干重/浮游藻类干 重; $a \geqslant 100$ 为草型稳态, $a \leqslant 1$ 为藻型稳态, $1<a<100$ 为过渡类型.

其中, 沉水植物干重生物量用沉水植物湿重生物量乘 0.08 表示 ${ }^{[17]}$, 藻类干重生物量通过 Chl. $a$ 浓度乘 70 再乘湖泊平均水深得到 ${ }^{[18]}$.

根据 Aizaki 修正的营养状态指数 (trophic state index, $T S I_{\mathrm{M}}$ ) 来评价研究水域的富营养化状态, 并用加权 平均计算处理,其中 Chl. $a$ 、TP 和透明度的比重分别为 $54 \% 、 29.7 \%$ 和 $16.3 \%$, 评价标准为: $T S I_{\mathrm{M}}<37$ 为贫营 养, $37 \leqslant T S I_{\mathrm{M}}<53$ 为中营养, $53 \leqslant T S I_{\mathrm{M}}<65$ 为富营养, $T S I_{\mathrm{M}} \geqslant 65$ 为重富营养 ${ }^{[19-21]}$.

\section{6 统计分析}

采用 One-way ANOVA 对不同区域光学特性和衰减因子的差异性进行分析. 线性回归运用 SPSS 20.0 进 行分析, 采用向后多元回归方法篮选不同区域内的主导衰减因子. 采用平均相对误差 (MAPE) 和均方根误差 $(R M S E)$ 两个指标对多元回归模型精度验证结果进行评价, 计算公式为:

$$
\begin{gathered}
M A P E=\frac{1}{n} \sum_{i=1}^{n}\left|\frac{E s_{i}-O_{i}}{O_{i}}\right| \\
R M S E=\sqrt{\frac{1}{n} \sum_{i=1}^{n}\left(E s_{i}-O_{i}\right)^{2}}
\end{gathered}
$$

式中, $n$ 为样点数目, $E s_{i}$ 和 $O_{i}$ 分别为第 $i$ 个样点的模型估算值和实测值.

\section{2 结果与分析}

\section{1 光合有效辐射垂向特征}

由于 PAR 测量数据较多, 因此在 3 种区域选择了 6 个采样点 $\left(1^{\#} 、 3^{\#} 、 4^{\#} 、 5^{\#} 、 6^{\#} 、 8^{\#}\right)$ 的 PAR 垂向分布特 征及指数回归结果进行展示 (图 2). 在现场测量过程中, 明显的异常值被舍弃, 重新进行测量. 各采样点的 指数拟合决定系数 $\left(R^{2}\right)$ 都达到了 0.9 以上, 其中, 无植物区域拟合效果较好, 沉水植物分布区域内拟合效果 较差, 测量时水下植物的遮挡是影响拟合效果的主要原因, 天气状况、波浪也可能对结果产生一定影响.
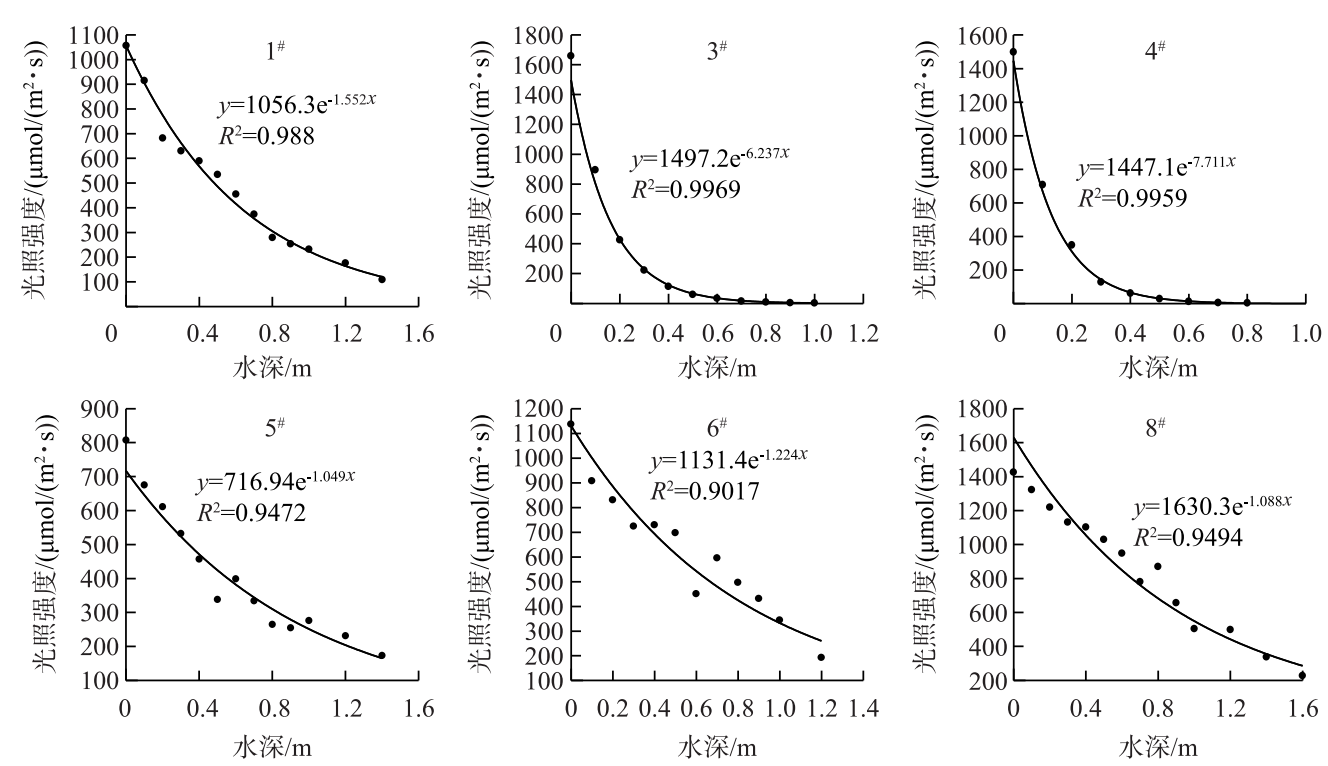

图 26 个代表点位 PAR 强度垂直分布特征

Fig.2 Vertical distribution characteristics of PAR intensity of six typical sampling sites 


\section{2 光学特性与影响因子的空间差异及线性拟合}

尽管东太湖面积很小,但不同区域 $k_{\mathrm{d}}(\mathrm{PAR})$ 的差异比较大 (表 1). 8 月份各采样点的 $k_{\mathrm{d}}(\mathrm{PAR})$ 均值在 1.04 6.35 $\mathrm{m}^{-1}$ 之间变化, 极差为 $5.31 \mathrm{~m}^{-1}$, 其中 $6^{\#} 、 7^{\#} 、 8^{\#}$ 点位 $k_{\mathrm{d}}(\mathrm{PAR})$ 均值较小, 范围为 $1.04 \sim 1.16 \mathrm{~m}^{-1}$, 该 区域为沉水植物茂盛区; $1^{\#} 、 2^{\#} 、 5^{\#} 、 10^{\#}$ 点位 $k_{\mathrm{d}}(\mathrm{PAR})$ 均值较大, 范围为 $1.39 \sim 3.47 \mathrm{~m}^{-1}$, 该区域为沉水植物稀 疏区; $3^{\#} 、 4^{\#} 、 9^{\#}$ 点位 $k_{\mathrm{d}}(\mathrm{PAR})$ 均值最大, 范围为 $5.97 \sim 6.35 \mathrm{~m}^{-1}$, 该区域为无植物区.

表 1 东太湖 $k_{\mathrm{d}}(\mathrm{PAR})$ 的计算结果

Tab.1 Results of $k_{\mathrm{d}}(\mathrm{PAR})$ values in eastern Lake Taihu

\begin{tabular}{|c|c|c|c|c|c|c|}
\hline \multirow{2}{*}{ 采样点 } & \multicolumn{4}{|c|}{$k_{\mathrm{d}}(\mathrm{PAR}) / \mathrm{m}^{-1}$} & \multirow{2}{*}{ 样本个数 $N$} & \multirow{2}{*}{ 所属区域 } \\
\hline & 均值 & 标准差 & 最大值 & 最小值 & & \\
\hline $1^{\#}$ & 1.80 & 0.248 & 2.55 & 1.55 & 16 & 稀疏区 \\
\hline $2^{\#}$ & 3.35 & 1.001 & 5.92 & 2.29 & 16 & 稀疏区 \\
\hline $3^{\#}$ & 5.97 & 1.270 & 9.64 & 4.41 & 16 & 无植物区 \\
\hline $4^{\#}$ & 6.35 & 1.796 & 11.80 & 4.66 & 16 & 无植物区 \\
\hline $5^{\#}$ & 1.39 & 0.470 & 2.82 & 1.01 & 16 & 稀疏区 \\
\hline $6^{\#}$ & 1.16 & 0.190 & 1.59 & 0.90 & 16 & 茂盛区 \\
\hline $7^{\#}$ & 1.04 & 0.330 & 1.89 & 0.73 & 16 & 茂盛区 \\
\hline $8^{\#}$ & 1.06 & 0.116 & 1.34 & 0.87 & 16 & 茂盛区 \\
\hline $9^{\#}$ & 6.25 & 1.453 & 8.92 & 4.71 & 16 & 无植物区 \\
\hline $10^{\#}$ & 3.47 & 0.817 & 5.49 & 1.90 & 16 & 稀疏区 \\
\hline
\end{tabular}

如图 3 所示, 东太湖 $k_{\mathrm{d}}(\mathrm{PAR})$ 的空间变化规律为: 植物茂盛区 $\left(1.09 \mathrm{~m}^{-1}\right)<$ 植物稀疏区 $\left(2.50 \mathrm{~m}^{-1}\right)<$ 无植物 区 $\left(6.19 \mathrm{~m}^{-1}\right) . k_{\mathrm{d}}(\mathrm{PAR})$ 在植物茂盛区最小, 变化范围为 $0.73 \sim 1.89 \mathrm{~m}^{-1}$, 平均值仅为 $1.09 \mathrm{~m}^{-1}$, 显著低于植物稀 疏区和无植物区 $(P<0.05) ; k_{\mathrm{d}}(\mathrm{PAR})$ 在无植物区最大, 变化范围为 $4.41 \sim 11.80 \mathrm{~m}^{-1}$, 平均值为 $6.19 \mathrm{~m}^{-1}$, 显著高 于植物茂盛区和植物稀疏区 $(P<0.05)$. 真光层深度与 $k_{\mathrm{d}}(\mathrm{PAR})$ 相反, 空间变化规律为无植物区 $(0.78 \mathrm{~m})<$ 植物 稀疏区 $(2.25 \mathrm{~m})<$ 植物茂盛区 $(4.38 \mathrm{~m})$, 变化范围为 $0.39 \sim 6.31 \mathrm{~m}, 3$ 种区域间存在显著性差异 $(P<0.05)$.
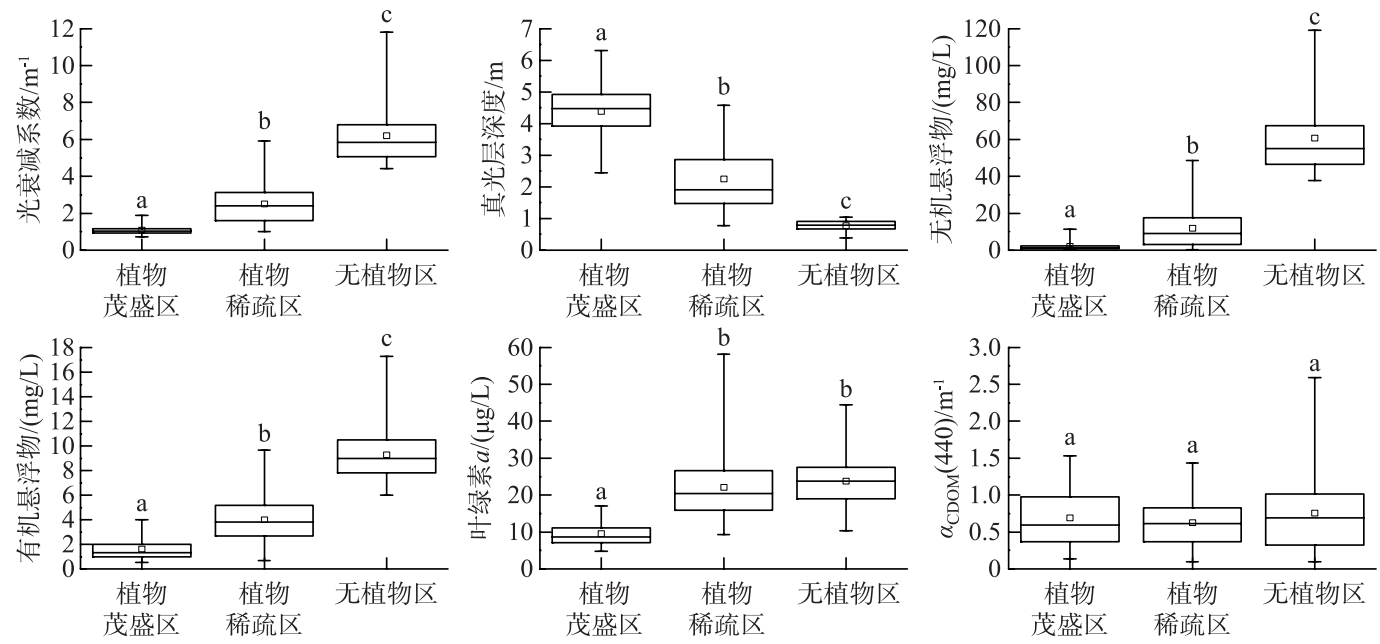

图 3 东太湖水体 $k_{\mathrm{d}}(\mathrm{PAR})$ 、真光层深度、ISS、OSS、叶绿素 $a$ 和 CDOM 吸收系数箱线图 (实线上、下端代表最大值和最小值; 矩形的上、下端代表上四分位数和下四分位数; 中间横线为中位线; 小正方形为均值; 图中字母表示 Tamhane's T2 多重比较检验结果, 字母不同表示差异显著 $(P<0.05))$

Fig.3 Box plot of $k_{\mathrm{d}}$ (PAR), euphotic depth, suspended particulate inorganic matter, suspended particulate organic matter, chlorophyll- $a$ and CDOM absorption coefficient in eastern Lake Taihu 
ISS 和 OSS 浓度的变化规律一致:植物茂盛区 ( ISS: $1.95 \mathrm{mg} / \mathrm{L}$, OSS: $1.63 \mathrm{mg} / \mathrm{L}$ ) <植物稀疏区 ( ISS: 11.91 $\mathrm{mg} / \mathrm{L}, \mathrm{OSS}: 4.00 \mathrm{mg} / \mathrm{L}$ ) <无植物区 ( ISS: $60.85 \mathrm{mg} / \mathrm{L}, \mathrm{OSS}: 9.29 \mathrm{mg} / \mathrm{L}$ ) , 二者浓度范围分别为 $0.33 \sim 119.27$ $\mathrm{mg} / \mathrm{L} 、 0.51 \sim 17.31 \mathrm{mg} / \mathrm{L}, 3$ 种区域间存在显著性差异 $(P<0.05)$; Chl. $a$ 浓度变化规律为植物茂盛区 (9.61 $\mu \mathrm{g} /$ $\mathrm{L})<$ 植物稀疏区 $(22.08 \mu \mathrm{g} / \mathrm{L})<$ 无植物区 $(23.74 \mu \mathrm{g} / \mathrm{L})$, 范围在 $4.79 \sim 58.21 \mu \mathrm{g} / \mathrm{L}$ 之间, 植物稀疏区与植物茂 盛区差异显著 $(P<0.05)$, 而与无植物区没有显著差异 $(P>0.05) ; \alpha_{\mathrm{CDOM}}(440)$ 变化规律为植物稀疏区 $(0.63$ $\left.\mathrm{m}^{-1}\right)<$ 植物茂盛区 $\left(0.69 \mathrm{~m}^{-1}\right)<$ 无植物区 $\left(0.76 \mathrm{~m}^{-1}\right)$, 变化范围为 $0.10 \sim 2.59 \mathrm{~m}^{-1}$, 且 3 种区域无显著性差异 $(P>0.05)$.

与无植物区相比, 沉水植物茂盛区 $k_{\mathrm{d}}$ (PAR) 减少了 $83.4 \%$, 光衰减因子中, ISS、OSS、Chl. $a$ 浓度和 $\alpha_{\mathrm{CDOM}}$ (440) 分别减少了 $96.8 \% 、 82.5 \% 、 59.5 \%$ 和 $9.2 \%$.

分别将整个东太湖和 3 种区域的 $k_{\mathrm{d}}$ (PAR) 与 ISS、OSS、Chl. $a$ 浓度和 CDOM 进行线性拟合, 结果发现东 太湖 $k_{\mathrm{d}}(\mathrm{PAR})$ 与 ISS 浓度有很好的线性关系, $R^{2}$ 为 0.947 (图 4a), 与 OSS 浓度的线性关系也比较好, $R^{2}$ 为 0.853 (图 4b), 但与 Chl. $a$ 浓度的线性关系较差, $R^{2}$ 为 0.151 (图 4c), 与 CDOM 吸收系数无显著线性关系, $R^{2}$ 为 0.022 (图 4d). 茂盛区 $k_{\mathrm{d}}(\mathrm{PAR})$ 与各影响因子均没有显著线性关系 (图 5); 稀疏区 $k_{\mathrm{d}}(\mathrm{PAR})$ 与 ISS、OSS 浓 度呈显著正线性关系, 与 Chl. $a$ 浓度、CDOM 吸收系数无显著线性关系 (图 6); 无植物区 $k_{\mathrm{d}}(\mathrm{PAR})$ 与 ISS、OSS 浓度呈显著正线性关系, 与 Chl. $a$ 浓度呈显著负线性关系, 与 CDOM 吸收系数无显著线性关系(图 7).
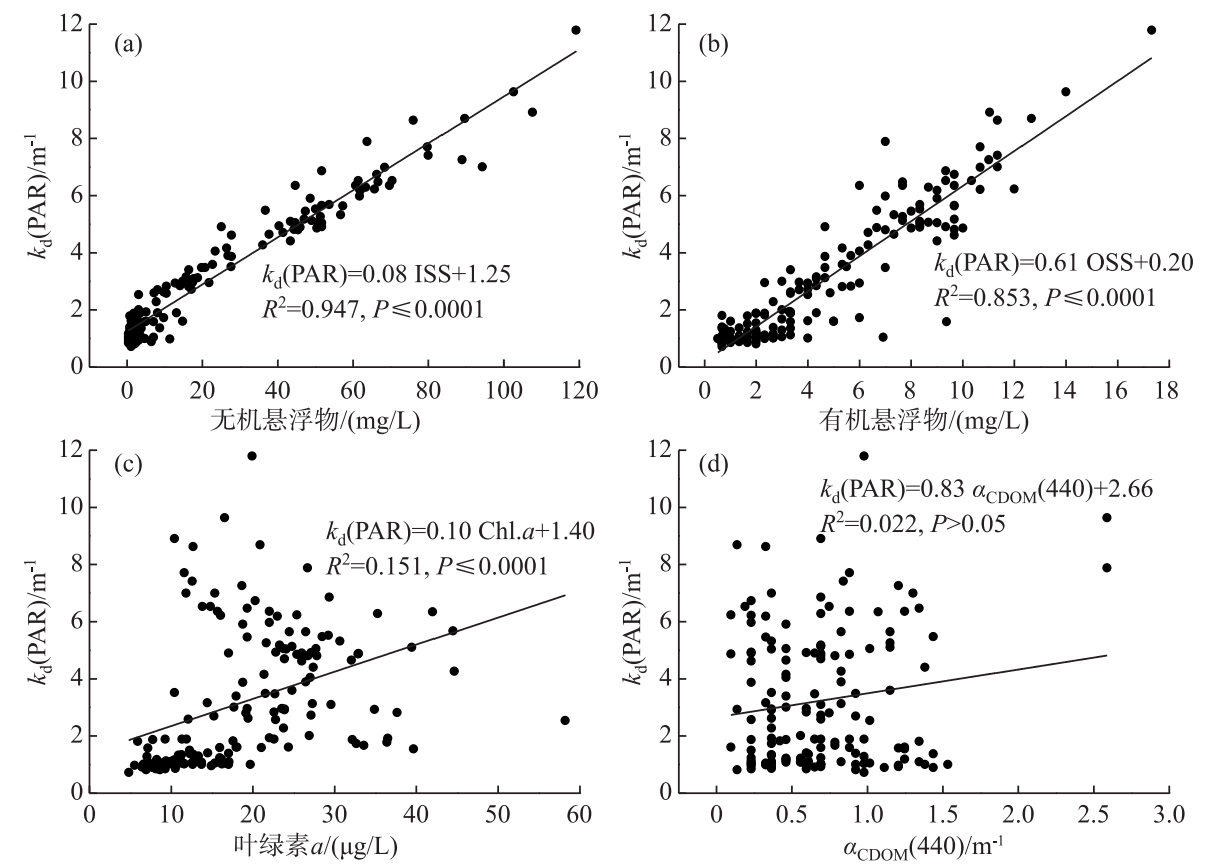

图 4 东太湖 $k_{\mathrm{d}}(\mathrm{PAR})$ 与无机悬浮物 (a)、有机悬浮物 (b)、叶绿素 $a(\mathrm{c})$ 和 CDOM 吸收系数 (d) 的线性拟合

Fig.4 Linear fitting between $k_{\mathrm{d}}(\mathrm{PAR})$ and suspended particulate inorganic matter (a), suspended particulate organic matter (b), chlorophyll-a (c) and CDOM absorption coefficient (d) in eastern Lake Taihu

\section{3 不同区域的主导影响因子}

上节分析表明, 东太湖不同区域的 $k_{\mathrm{d}}(\mathrm{PAR})$ 差异显著, 衰减因子也有较大的区别. 通过向后法进行多元 线性回归, 对每种区域光衰减的主导因子进行分析. 沉水植物茂盛区回归模型先后剔除了 $\alpha_{\mathrm{CDOM}}(440)$ 、 Chl. $a$ 、ISS 和 OSS 浓度, 表明影响该区域水下光衰减的因素主要是水体本身的吸收和散射; 沉水植物稀疏区 的 $k_{\mathrm{d}}(\mathrm{PAR})$ 与衰减因子的表达式见表 2 模型 1 , 模型先后剔除了 OSS 浓度和 $\alpha_{\mathrm{CDOM}}(440)$, 保留了 ISS 和Chl. $a$ 浓度, 说明该区域水下光照衰减由 ISS 和 Chl. $a$ 浓度共同主导, 模型精度较高, 拟合结果 $R^{2}$ 为 0.883 , 验证结 

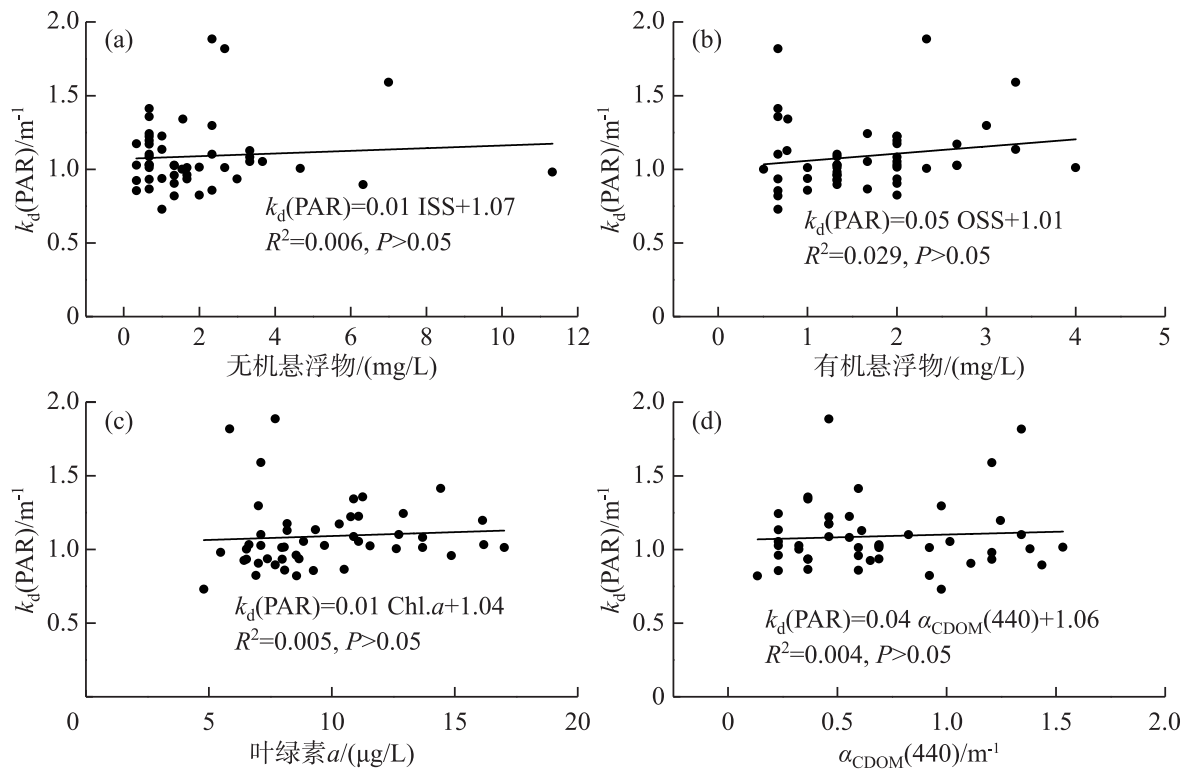

图 5 植物茂盛区 $k_{\mathrm{d}}(\mathrm{PAR})$ 与无机悬浮物 $(\mathrm{a})$ 、有机悬浮物 $(\mathrm{b})$ 、叶绿素 $a(\mathrm{c})$ 和 CDOM 吸收系数 $(d)$ 的线性拟合

Fig.5 Linear fitting between $k_{\mathrm{d}}(\mathrm{PAR})$ and suspended particulate inorganic matter (a), suspended particulate organic matter (b), chlorophyll-a (c) and CDOM absorption coefficient (d) in submerged plants sparse region
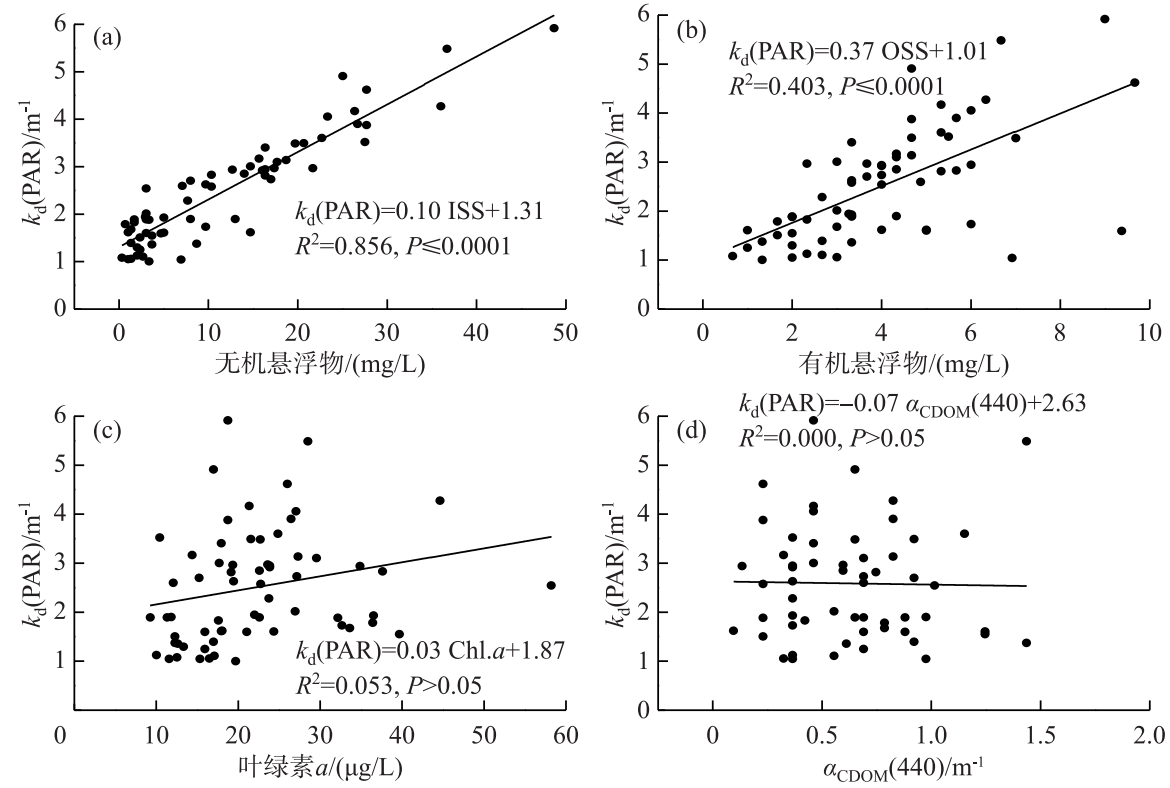

图 6 植物稀疏区 $k_{\mathrm{d}}(\mathrm{PAR})$ 与无机悬浮物 ( a )、有机悬浮物 $(\mathrm{b})$ 、 叶绿素 $a(\mathrm{c})$ 和 CDOM 吸收系数 (d) 的线性拟合

Fig.6 Linear fitting between $k_{\mathrm{d}}(\mathrm{PAR})$ and suspended particulate inorganic matter (a), suspended particulate organic matter (b), chlorophyll- $a$ (c) and CDOM absorption coefficient (d) in submerged plants lush region 

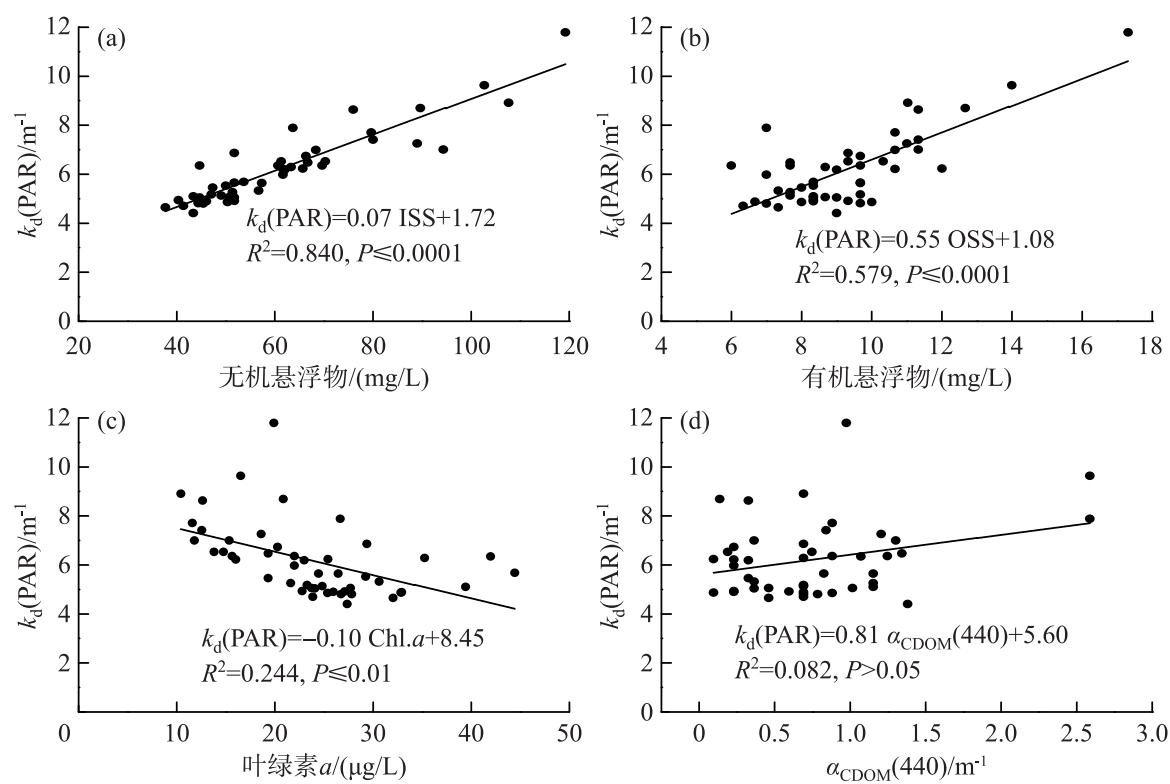

图 7 无植物区 $k_{\mathrm{d}}(\mathrm{PAR})$ 与无机悬浮物 (a)、有机悬浮物 ( $\mathrm{b}$ )、叶绿素 $a(\mathrm{c})$ 和 CDOM 吸收系数 (d) 的线性拟合

Fig.7 Linear fitting between $k_{\mathrm{d}}(\mathrm{PAR})$ and suspended particulate inorganic matter (a), suspended particulate organic matter (b), chlorophyll-a (c) and CDOM absorption coefficient (d) in no-plant region

果 MAPE 和 RMSE 分别为 $15.8 \%$ 和 0.412 ; 表 2 中模型 2 为无植物区域的 $k_{\mathrm{d}}(\mathrm{PAR})$ 回归方程,模型先后剔除 了 OSS 浓度、 $\alpha_{\mathrm{CDOM}}(440)$ 和 Chl. $a$ 浓度, 仅保留了 ISS 浓度, 因此, 该区域的 ISS 浓度是水下光照衰减的主导 因子, 模型精度也比较高, 拟合结果 $R^{2}$ 为 0.840 , 验证结果 MAPE 和 $R M S E$ 分别为 $6.3 \%$ 和 0.636 .

表 2 多元线性回归模型验证结果的误差统计

Tab.2 Error statistics of validation results for multiple linear regression models

\begin{tabular}{ccccc}
\hline 模型 & 多元回归方程 & $R^{2}$ & $M A P E / \%$ & $R M S E$ \\
\hline 1 & $k_{\mathrm{d}}(\mathrm{PAR})=0.10$ ISS +0.01 Chl. $a+1.06$ & 0.883 & 15.8 & 0.412 \\
2 & $k_{\mathrm{d}}(\mathrm{PAR})=0.07$ ISS+1.71 & 0.840 & 6.3 & 0.636 \\
\hline
\end{tabular}

\section{4 东太湖草藻干重比和区域富营养化评价}

表 3 记录了采样期间各采样点沉水植物生物量和优势种, 根据沉水植物生物量和叶绿素 $a$ 浓度数据, 计算出各采样点草藻干重比, 见表 4. 采用 $T S I_{\mathrm{M}}$ 方法评价区域富营养化状态, 见表 5. 总体上讲, 东太湖目前 处于由草型湖泊向藻型湖泊过渡的阶段, 并且湖泊处于富营养水平, 相比以前的草型稳态 ${ }^{[22]}$, 东太湖面临着 向藻型稳态转变的趋势. 其中, 沉水植物茂盛区草藻干重比大于 100 , 属于草型稳态, 区域处于中营养水平; 稀疏区草藻干重比在 $1 \sim 100$ 之间, 属于草藻共生状态, 区域处于富营养水平; 无植物区位于行洪通道区域, 没有沉水植物分布, 属于藻型稳态, 处于重富营养水平.

\section{3 讨论}

东太湖面积虽小,但本研究发现, 不同区域的 $k_{\mathrm{d}}$ (PAR) 存在显著性差异, 各采样点均值在 $1.04 \sim 6.35 \mathrm{~m}^{-1}$ 之间变化. 其中沉水植物分布茂盛的区域 $k_{\mathrm{d}}(\mathrm{PAR})$ 最小, 主要是因为沉水植物一方面可以减小波浪的冲刷 作用、防止底泥县浮、降低水体中的悬浮物浓度, 另一方面沉水植物能够分泌化感物质抑制藻类的生长, 降 低 Chl. $a$ 浓度 ${ }^{[23-24]}$. 采样期间台风对该区域基本没有影响, $k_{\mathrm{d}}$ (PAR) 变化不明显. 行洪通道区域 $k_{\mathrm{d}}(\mathrm{PAR})$ 最 
表 3 沉水植物生物量和优势种

Tab.3 Biomass and the dominant species of submerged plants

\begin{tabular}{|c|c|c|c|c|}
\hline 区域类型 & 采样点 & $\begin{array}{c}\text { 沉水植物湿重生物量/ } \\
\left(\mathrm{kg} / \mathrm{m}^{2}\right)\end{array}$ & $\begin{array}{l}\text { 均值/ } \\
\left(\mathrm{kg} / \mathrm{m}^{2}\right)\end{array}$ & 优势种 \\
\hline \multirow[t]{3}{*}{ 沉水植物茂盛区 } & $6^{\#}$ & 2.18 & 2.31 & 金鱼藻 (Ceratophyllum demersum) \\
\hline & $7^{\#}$ & 2.29 & & 金鱼藻、黑藻 (Hydrilla verticillata) \\
\hline & $8^{\#}$ & 2.46 & & 黑藻 \\
\hline \multirow[t]{4}{*}{ 沉水植物稀疏区 } & $1^{\#}$ & 0.23 & 0.78 & 金鱼藻 \\
\hline & $2^{\#}$ & 1.22 & & 金鱼藻、黑藻 \\
\hline & $5^{\#}$ & 1.21 & & 黑藻 \\
\hline & $10^{\#}$ & 0.47 & & 金鱼藻、黑藻 \\
\hline
\end{tabular}

表 4 东太湖各采样点草藻干重比

Tab.4 Ratio of submerged plant biomass to algae biomass at each sampling site in eastern Lake Taihu

\begin{tabular}{|c|c|c|c|c|c|c|c|c|c|c|c|}
\hline \multirow{2}{*}{ 指标 } & \multicolumn{3}{|c|}{ 沉水植物茂盛区 } & \multicolumn{4}{|c|}{ 沉水植物稀疏区 } & \multicolumn{3}{|c|}{ 无植物区 } & \multirow{2}{*}{ 均值 } \\
\hline & $6^{\#}$ & $7^{\#}$ & $8^{\#}$ & $1^{\#}$ & $2^{\#}$ & $5^{\#}$ & $10^{\#}$ & $3^{\#}$ & $4^{\#}$ & $9^{\#}$ & \\
\hline 沉水植物干重生物量 $/\left(\mathrm{kg} / \mathrm{m}^{2}\right)$ & 0.17 & 0.18 & 0.20 & 0.02 & 0.10 & 0.097 & 0.04 & 0 & 0 & 0 & 0.08 \\
\hline 叶绿素 $a /(\mu \mathrm{g} / \mathrm{L})$ & 9.72 & 7.16 & 11.96 & 28.46 & 22.73 & 14.13 & 23.00 & 25.05 & 22.73 & 23.44 & 18.84 \\
\hline 草藻干重比 & 135.05 & 194.26 & 129.17 & 5.43 & 33.98 & 54.63 & 13.43 & 0 & 0 & 0 & 56.59 \\
\hline
\end{tabular}

表 53 种区域富营养化指标值及评价结果

Tab.5 Values of water quality indexes and assessment results of eutrophication for three regions

\begin{tabular}{ccccccccc}
\hline 区域类型 & $\begin{array}{c}\text { 叶绿素 } a / \\
(\mu \mathrm{g} / \mathrm{L})\end{array}$ & $\begin{array}{c}\text { 总磷/ } \\
(\mathrm{mg} / \mathrm{L})\end{array}$ & $\begin{array}{c}\text { 透明度/ } \\
\mathrm{m}\end{array}$ & $\begin{array}{c}T S I_{\mathrm{M}} \\
(\mathrm{Chl} . a)\end{array}$ & $\begin{array}{c}T S I_{\mathrm{M}} \\
(\mathrm{TP})\end{array}$ & $\begin{array}{c}T S I_{\mathrm{M}} \\
(\mathrm{SD})\end{array}$ & $T S I_{\mathrm{M}}$ & 等级 \\
\hline 茂盛区 & 9.61 & 0.019 & 1.65 & 49.3 & 48.2 & 56.5 & 51.3 & 中营养 \\
稀疏区 & 21.51 & 0.043 & 0.76 & 58.1 & 58.2 & 69.5 & 61.5 & 富营养 \\
无植物区 & 23.74 & 0.071 & 0.20 & 59.2 & 64.6 & 91.7 & 69.7 & 重富营养 \\
均值 & 18.29 & 0.044 & 0.87 & 55.5 & 57.0 & 72.6 & 60.8 & 富营养 \\
\hline
\end{tabular}

大, 区域内没有沉水植物, 流速和波浪比较大, 水体浑浊. 该区域承接大太湖的流水, 水体悬浮物浓度比较 高, 再加上风浪的扰动, 悬浮物沉降较慢, 风浪较大时底泥还会悬浮, 因此悬浮物浓度最高. 沉水植物相对稀 疏的区域基本位于围网拆除区域内部, 其中 $1^{\#}$ 点在东山沿岸附近, 该区域的 $k_{\mathrm{d}}(\mathrm{PAR})$ 较大. 围网拆除区域的 点位会受行洪通道区域的影响, 中心区域波浪较大时会向两边传播, 虽然沉水植物可以固着底泥, 但波浪引 起的切应力大于底泥起动应力时就会造成底泥的再悬浮, 台风过后可以观察到水体悬浮物浓度明显增加.

虽然 $k_{\mathrm{d}}(\mathrm{PAR})$ 属于表观光学特性, 但是主要由固有光学特性决定, 尽管它受到人射光场变化的影响, 但 并不十分敏感 ${ }^{[25]}$. 张运林等 ${ }^{[26]}$ 通过在不同表面光强下得出的光学衰减系数分析发现, 其基本上与表面光强 无关, 不随光场条件的改变而改变, 主要受制于水中的组成物质. 不同湖泊光衰减的主要因素也有区别. van Duin 等估算了荷兰 20 个浅水湖泊中各成分对衰减系数的贡献, 发现在大多数湖泊, 光衰减主要是由藻类生 物量引起的 ${ }^{[27]}$. 美国佛罗里达洲的一个大型富营养化浅水湖泊 Apopka 湖中, Chl. $a$ 对光衰减的贡献率甚至 超过 50\%. Erikson 等研究发现 Xolotlán 湖的水下光照深度主要受 Chl. $a$ 浓度的调节 ${ }^{[28]}$. 还有一些研究认为 悬浮物浓度是影响光衰减的重要因素 ${ }^{[29-30]}$.

然而我们的研究发现, 东太湖不同区域的主导衰减因子有所不同. 在沉水植物分布茂盛的区域, 光合有 效辐射衰减系数模型剔除了所有变量, 区域内光衰减系数较小, 主要是水体本身对光的吸收和散射. 在沉水 
植物分布稀疏的区域, ISS 和 Chl. $a$ 浓度较高, 二者共同主导了光照的衰减, 该区域属于草藻共生状态. 虽然 也有沉水植物分布, 但是生物量比较小, 对底泥和藻类的抑制作用相对减弱, 在外力的扰动下容易发生底泥 悬浮, 释放营养盐, 为藻类生长提供营养物质. 以往对太湖的研究表明, 水下光照衰减主要由悬浮物引 起 ${ }^{[11,30]}$, 但我们对东太湖沉水植物稀疏区域的研究发现, Chl. $a$ 也是影响水下光照衰减的重要因素之一, 在 生态修复实践中不容忽视. 近几年沉水植物的逐渐减少表明东太湖目前可能接近稳定转变的关键阶段, 从 大型水生植物主导的清澈状态向浮游植物主导的浑浊状态转变将导致许多浅水湖泊水质持续不良, 然而, 一旦转变后再恢复大型水生植物主导的清澈状态往往更加困难 ${ }^{[9]}$. 因此需要重点关注此区域, 减小波浪对 底泥扰动造成的底泥悬浮和营养盐释放, 降低湖水中悬浮物和 Chl. $a$ 浓度. 在无植物区域, ISS 浓度要比 Chl. $a$ 浓度大得多, 是影响光衰减的主要因素. 该区域底泥容易再悬浮, 在水流的输移作用下泥沙会向植物 区输送, 进而影响到两侧沉水植物的生长. 3 个区域的 OSS 和 CDOM 影响都比较小, 一方面是因为东太湖 OSS 和 CDOM 浓度比较低, 悬浮物主要由 ISS 构成, OSS 仅占到总悬浮物的 $17 \%$, 导致引起水下光照衰减的 影响无法显现, 另一方面 CDOM 对光的吸收在 $400 \mathrm{~nm}$ 以下的紫外短波部分贡献比较高, $500 \mathrm{~nm}$ 以上的光衰 减主要来源于悬浮物和 Chl. $a$.

真光层深度是湖泊物理、化学、生物综合作用的关键指标, 在湖泊生态修复和水质改善方面发挥着重要 作用 ${ }^{[31]}$. 真光层深度与水深的比值可以作为划分是否可恢复沉水植物的标准, 比值大于 1 时, 表明水下光照 环境可满足沉水植物的生长需求. 根据真光层深度与透明度的关系, 在实际测量中可以通过测量湖水透明 度来判断区域内沉水植物能否生存. 本文根据计算的真光层深度和测量的透明度数据, 建立了回归方程, 结 果表明在置信区间为 $95 \%$ 的水平下, 真光层深度和水体透明度呈显著的正线性关系: $D_{\mathrm{eu}}=2.70 S D\left(R^{2}=\right.$ $0.969, P \leqslant 0.0001, n=160)$. 真光层深度大约是透明度的 2.7 倍, 在实际应用中, 可通过测量透明度反算出 真光层深度, 然后比较真光层深度与水深的大小, 当真光层深度 $\geqslant$ 水深时, 表明可以恢复沉水植物. 采样期 间东太湖平均水深 $1.85 \mathrm{~m}$, 即将透明度提高到 $0.7 \mathrm{~m}$ 以上, 就有可能恢复沉水植物, 因此当地生态修复工程 中可借鉴此数据. 总的来说, 东太湖光照衰减的主要因素是 ISS 和 Chl. $a$, 水下光照的分布决定了沉水植物的 分布情况. 目前, 东太湖正处于从草型湖区到藻型湖区过渡的阶段, 尤其在沉水植物稀疏区域, 围网拆除后 增加的水动力对沉水植物生长造成了不利影响. 另外, 该区域长有许多浮叶植物, 阻碍了光照在水下的传 播, 进一步影响了沉水植物的光照需求. 因此, 建议可采取围隔等消浪措施减小水动力, 防止底泥悬浮, 提高 水体透明度, 同时加强水生植物管理, 适度收割浮叶植物, 改善水下光照环境, 保证沉水植物生长良好, 促进 湖水水体的良性循环. 鉴于大太湖藻类输人的影响, 有关部门需要及时关注和监测东太湖人口处一一东荠 嘴附近的水质情况, 采取相关措施阻止外源性藻类入侵东太湖, 削弱藻类与沉水植物的竞争力.

\section{4 结论}

1) 东太湖 $k_{\mathrm{d}}(\mathrm{PAR})$ 范围为 $0.73 \sim 11.8 \mathrm{~m}^{-1}$, 空间变化规律为: 植物茂盛区 $\left(1.09 \mathrm{~m}^{-1}\right)<$ 植物稀疏区 $(2.50$ $\left.\mathrm{m}^{-1}\right)<$ 无植物区 $\left(6.19 \mathrm{~m}^{-1}\right)$, 对应的真光层深度范围为 $0.39 \sim 6.31 \mathrm{~m}$. 东太湖光学特性空间差异显著. 衰减因 子中, ISS 和 OSS 浓度空间差异显著 $(P<0.05)$, CDOM 吸收系数无显著性差异 $(P>0.05)$, 沉水植物稀疏区叶 绿素 $a$ 浓度显著高于植物茂盛区 $(P<0.05)$, 但与无植物区没有显著差异 $(P>0.05)$. 沉水植物的分布影响着 区域内的水质因子变化.

2) $k_{\mathrm{d}}(\mathrm{PAR})$ 与 ISS、OSS 浓度的线性拟合较好, 与 Chl. $a 、$ CDOM 拟合较差; 沉水植物茂盛区衰减主要是水 体本身对光的吸收和散射作用, 稀疏区属于草藻共生状态, 由 Chl. $a$ 和 ISS 共同主导, 无植物区域主导衰减 因子为 ISS, 整个区域的 OSS 和 CDOM 对光照衰减基本无影响. ISS 和 Chl. $a$ 浓度是影响该区域光照衰减的 主要因子.

3) 东太湖正处于由草型稳态向藻型稳态过渡的阶段, 整个湖泊属于富营养水平, 其中沉水植物茂盛区 属于草型稳态, 处于中营养水平; 稀疏区属于草藻共生状态, 处于富营养水平; 无植物区属于藻型稳态, 处于 重富营养水平.

4) 东太湖真光层深度大约是透明度的 2.7 倍. 


\section{5 参考文献}

[ 1 ] Piepho M. Assessing maximum depth distribution, vegetated area, and production of submerged macrophytes in shallow, turbid coastal lagoons of the southern Baltic Sea. Hydrobiologia, 2017, 794(1) : 303-316. DOI: 10.1007/s10750-0173107-5.

[ 2 ] Wang H, Pang Y, Liu SB et al. Research progress on influencing of environmental factors on the growth of submersed macrophytes. Acta Ecologica Sinica, 2008, 28(8):3958-3968. [王华, 逢勇, 刘申宝等. 沉水植物生长影响因子研究进 展. 生态学报, 2008, 28(8) : 3958-3968.]

[ 3 ] Kirk JTO, Press C. Light \& photosynthesis in aquatic ecosystems. Journal of Ecology, 1983, 45 ( 3 ). DOI: 10. 1017/CBO9780511623370

[ 4 ] di Toro DM. Optics of turbid estuarine waters: Approximations and applications. Water Research, 1978, 12(12) : 10591068. DOI: 10.1016/0043-1354(78)90051-9.

[ 5 ] Yang P, Gao XY, Cai QM. Measurement of optical properties of soluble organic materials suspended particles and pigment of plankton algae sampled from Taihu Lake. Transaction of Oceanology and Limnology, 1992, (1): 63-71. [ 杨平, 高锡 芸, 蔡启铭. 太湖水中可溶性有机质, 悬浮质和浮游藻类色素的光学特性的测定. 海洋湖沼通报, 1992, (1): 63-71.]

[ 6 ] Yang DT, Chen WM, Zhang YL et al. Effect of underwater light spectrum on primary production of the Taihu Lake. Journal of Ecology and Rural Environment, 2003, 19(2) : 24-28. [杨顶田, 陈伟民, 张运林等. 太湖水体光学特征及其对 水中初级生产力的影响. 生态与农村环境学报, 2003, 19(2): 24-28.]

[ 7 ] Zhang YL, Qin BQ, Chen WM et al. Analysis on distribution and variation of beam attenuation coefficient of Taihu Lake's water. Advances in Water Science, 2003, 14(4): 447-453. [张运林, 秦伯强, 陈伟民等. 太湖水体光学衰减系数的分 布及其变化特征. 水科学进展, 2003, 14(4): 447-453.]

[ 8 ] Gu XH, Zhang SZ, Bai XL et al. Evolution of community structure of aquatic macrophytes in east Taihu Lake and its wetlands. Acta Ecologica Sinica, 2005, 25(7) : 1541-1548. [谷孝鸿, 张圣照, 白秀玲等. 东太湖水生植物群落结构的 演变及其沼泽化. 生态学报, 2005, 25(7) : 1541-1548.]

[ 9 ] Zhang YL, Liu XH, Qin BQ et al. Aquatic vegetation in response to increased eutrophication and degraded light climate in eastern Lake Taihu: Implications for lake ecological restoration. Scientific Reports, 6(1): 23867. DOI: 10. 1038/ srep23867.

[10] Zhao K, Zhou YF, Jiang ZL et al. Changes of aquatic vegetation in Lake Taihu since 1960s. J Lake Sci, 2017, 29(2): 351-362. DOI: 10.18307/2017.0211. [赵凯, 周彦锋, 蒋兆林等. 1960 年以来太湖水生植被演变. 湖泊科学, 2017, 29(2) : 351-362.]

[11] Zhang YL, Feng S, Ma RH et al. Spatial pattern of euphotic depth and estimation of phytoplankton primary production in Lake Taihu in autumn 2004. J Lake Sci, 2008, 20(3) : 380-388. DOI: 10.18307/2008.0319. [张运林, 冯胜, 马荣华 等. 太湖秋季真光层深度空间分布及浮游植物初级生产力的估算. 湖泊科学, 2008, 20(3) : 380-388.]

[12] Gu XH, Wang XR, Hu WP. Effect of fishery development on water environment and its eco-countermeasure in east Lake Taihu. Shanghai Environmental Sciences, 2003, 22(10): 702-704, 711. [谷孝鸿, 王晓蓉, 胡维平. 东太湖渔业发展 对水环境的影响及其生态对策. 上海环境科学, 2003, 22(10): 702-704, 711.]

[13] Jin XC, Tu QY eds. Specifications for lake eutrophication survey: 2nd Edition. Beijing: China Environment Science Press, 1990. [ 金相灿, 屠清瑛. 湖泊富营养化调查规范. 北京: 中国环境科学出版社, 1990. ]

[14] Bricaud A, Morel A, Prieur L. Absorption by dissolved organic matter of the sea (yellow substance) in the UV and visible domains. Limnology and Oceanography, 1981, 26(1) : 43-53. DOI: 10.4319/lo.1981.26.1.0043.

[15] Zhongping LE. KPAR: An optical property associated with ambiguous values. J Lake Sci, 2009, 21(2) : 159-164. DOI: 10.18307/2009.0202. [李忠平. KPAR:一个数值模糊的光学量. 湖泊科学, 2009, 21(2): 159-164.]

[16] Bachmann RW, Horsburgh CA, Hoyer MV et al. Relations between trophic state indicators and plant biomass in Florida lakes. Hydrobiologia, 2002, 470(1/2/3) : 219-234. DOI: 10.1023/ A: 1015660922494.

[17] Chen HD, He CH. Standing crop of the macrophytes of lake tung-hu, Wuchang, with reference to the problem of its rational piscicultural utilization. Acta Hydrobiologica Sinica, 1975, 5(3) : 410-420. [陈洪达, 何楚华. 武昌东湖水生维管束 
植物的生物量及其在渔业上的合理利用问题. 水生生物学集刊, 1975, 5(3): 410-420.]

[18] Scheffer M ed. The limits of knowledge. Ecology of shallow lakes. Dordrecht: Springer Netherlands, 2004: 308-313. DOI: 10.1007/978-1-4020-3154-0_7.

[19] Aizaki M, Otsuki A, Fukushima T et al. Application of Carlson's trophic state index to Japanese lakes and relationships between the index and other parameters. Proceedings-International Association of Theoretical and Applied Limnology, 1981, 16(1) : 19-22. DOI: $10.1145 / 15691.15697$.

[20] Cai QH, Hu ZY. Studies on eutrophication problem and control strategy in the Three Gorges reservoir. Acta Hydrobiologica Sinica, 2006, 30 (1) : 7-11. [蔡庆华, 胡征宇. 三峡水库富营养化问题与对策研究. 水生生物学报, 2006, 30(1): 7-11.]

[21] Cai QH, Liu JK, Lorenz K. A comprehensive model for assessing lake eutrophication. Chinese Journal of Applied Ecology, 2002, 13(12) : 1674-1678. [ 蔡庆华, 刘建康, Lorenz King. 评价湖泊富营养化的一个综合模型 (英文). 应用生态 学报, 2002, 13(12): 1674-1678.]

[22] Li WC. Multiplicity of stable states in shallow lakes and its application. J Lake Sci, 1997, 9(2): 97-104. DOI: 10. 18307/1997.0201. [李文朝. 浅水湖泊生态系统的多稳态理论及其应用. 湖泊科学, 1997, 9(2) : 97-104.]

[23] Tong CH, Yang XE, Pu PM. Effects and mechanism of hydrophytes on control of release of nutrient salts in lake sediment. Journal of Agro-Environment Science, 2003, 22(6) : 673-676. [童昌华, 杨肖娥, 誉培民. 水生植物控制湖泊底泥营 养盐释放的效果与机理. 农业环境科学学报, 2003, 22(6): 673-676.]

[24] Xian QM, Chen HD, Zou HX et al. Allelopathic effects of four submerged macrophytes on Microcystis aeruginosa. J Lake Sci, 2005, 17 (1) : 75-80. DOI: 10.18307/2005.0112. [鲜啟鸣, 陈海东, 邹惠仙等. 四种沉水植物的克藻效应. 湖 泊科学, 2005, 17(1): 75-80.]

[25] Kirk JTO. Effects ofsuspensoids (turbidity) on penetration of solar radiation in aquatic ecosystems. Hydrobiologia, 1985, 125(1) : 195-208. DOI: 10.1007/BF00045935.

[26] Zhang YL, Qin BQ, Chen WM et al. Experimental study on underwater light intensity and primary productivity caused by variation of total suspended matter. Advances in Water Science, 2004, 15(5) : 615-620. [张运林, 秦伯强, 陈伟民等. 悬 浮物浓度对水下光照和初级生产力的影响. 水科学进展, 2004, 15(5) : 615-620.]

[27] vanDuin EHS, Blom G, Los FJ et al. Modeling underwater light climate in relation to sedimentation, resuspension, water quality and autotrophic growth. Hydrobiologia, 2001, 444: 25-42. DOI: 10.1023/ A: 1017512614680.

[28] Erikson R, Hooker E, Mejia M et al. Optimal conditions for primary production in a polymictic tropical lake ( Lake Xolotlán, Nicaragua). Hydrobiologia, 1998, 382(1) : 1-16. DOI: 10.1023/A:1003271614344.

[29] Lawson SE, Wiberg PL, McGlathery KJ et al. Wind-driven sediment suspension controls light availability in a shallow coastal lagoon. Estuaries and Coasts, 2007, 30(1) : 102-112. DOI: 10.1007/BF02782971.

[30] Zhang YL, Qin BQ, Chen WM et al. Regression analysis of beam attenuation coefficient under water in Lake Taihu. Oceanologia et Limnologia Sinica, 2004, 35(3) : 209-213. [张运林, 秦伯强, 陈伟民等. 太湖水体光学衰减系数的 特征及参数化. 海洋与湖沼, $2004,35(3): 209-213$.

[31] Zhang YL, Qin BQ, Hu WP et al. Temporal and spatial variation of the euphotic zone depth and its ecological significance in typical lake areas of Lake Taihu. Science in China: Series D, 2006, 36(3) : 287-296. [张运林, 秦伯强, 胡维平等. 太湖典型湖区真光层深度的时空变化及其生态意义. 中国科学 : 地球科学, 2006, 36(3) : 287-296. ] 